\title{
Damage Thresholds at Metal Surfaces for Short Pulse IR Lasers
}

\author{
JOSEPH F. FIGUEIRA AND SCOTT J. THOMAS
}

\begin{abstract}
Measurements of damage to metal surfaces induced by intense nanosecond pulses of IR radiation are reported. Single-shot damage thresholds of $\mathrm{Cu}$, stainless steel, molybdenum, and aluminum surfaces have been measured for various angles of incidence and the predicted increase in damage thresholds for grazing incidence optical components have been experimentally verified for the first time at $10 \mu \mathrm{m}$. In addition multiple-shot damage tests have been performed and practical lifetime curves for $\mathrm{Cu}$ mirrors have been established. The results are compared with existing theoretical models and shown to be in general agreement.
\end{abstract}

\section{INTRODUCTION}

$\mathbf{I}^{\mathrm{N}}$ $\mathrm{N}$ the design and operation of high-power IR lasers, the constraints imposed by damage limitations to the optical components of the system have a major impact on the cost, reliability, and power capability of the device. Previous work by several authors has documented the damage thresholds of various materials used in high power [1], [2] $\mathrm{CO}_{2}$ lasers. Recently, multiple-shot measurements of damage thresholds have been reported [1], [3]. In this paper, we will consider the general topic of damage induced in metal surfaces by intense, short-pulse $(1 \mathrm{~ns}) \mathrm{CO}_{2}$ lasers. The results of normal incidence single-shot damage threshold measurements in metals will be described and the experimental results will be compared to existing models for surface melting. The results of extended lifetime tests for $\mathrm{Cu}$ mirrors will be discussed and lifetime curves for $\mathrm{Cu}$ mirrors will be established. Finally, the use of metal mirror surfaces at grazing incidence angles will be discussed and damage threshold measurements will be presented and compared to the Fresnel theory for metal surface absorption at nonnormal incidence angles.

\section{Normal Incidence Damage to Metal Surfaces}

When an intense beam of light interacts with a metal surface normal to the beam direction, a fraction of the incident radiation penetrates the metal to a skin depth and is absorbed by the free carriers in the metal. If the absorption is strong enough and the incident radiation intense enough, the absorbed energy can raise the surface temperature to a value in excess of the melting temperature. When this occurs, permanent structural changes in the metal surface can take place [4], [5]. If this surface is used as an optical component, these changes can modify or degrade its intended performance. In this section we will discuss measurements and calculations of damage to

\footnotetext{
Manuscript received May 29, 1981 ; revised April 12, 1982. This work was performed under the auspices of the U.S. Department of Energy.

The authors are with Los Alamos National Laboratory, University of California, Los Alamos, NM 87545.
}

optical quality surfaces. We will briefly review the current theory of optical damage and describe experimental results for normal-incidence single-shot damage thresholds for a variety of metal surfaces and several pulse durations. As a final point in this section we will describe the results of multishot damage measurements to mirror surfaces and will deduce performance criteria for $\mathrm{Cu}$ mirrors.

Establishing a unique criterion for damage in a metal surface has proven difficult because of the variety of damage mechanisms proposed and observed. For the single-shot damage threshold measurements, we have chosen to define surface damage as any change in the mirror surface that is visually observable under low power microscopic examination (see below). Several authors have attempted to relate these observed surface effects to the actual physical processes occurring in the metal surfaces. Thresholds for surface melting have been derived previously for the case of constant surface absorption [6], [7] and have shown that for a pulse of intensity $I /\left(\mathrm{W} / \mathrm{cm}^{2}\right)$ and duration $t_{p}(\mathrm{~s})$

$$
\left(I t_{p}\right)_{m}=\frac{T_{m}-T_{o}}{2 A_{o}} \sqrt{\pi K C t_{p}}
$$

where $\left(I t_{p}\right)_{m}$ is the fluence $\left(\mathrm{J} / \mathrm{cm}^{2}\right)$ required to melt the surface, $A_{o}$ is the room temperature surface absorption, $T_{m}$ is the melting temperature for the material $\left({ }^{\circ} \mathrm{C}\right), T_{o}$ is the ambient temperature $\left({ }^{\circ} \mathrm{C}\right), K$ is the thermal conductivity in $\left(\mathrm{W} /\left(\mathrm{cm}^{2} \cdot{ }^{\circ} \mathrm{C}\right)\right.$ ), and $C$ is the volumetric specific heat (specific heat $x$ density) in $\left(\mathrm{W} \cdot \mathrm{s} /\left(\mathrm{cm}^{3} \cdot{ }^{\circ} \mathrm{C}\right)\right)$.

In general the surface absorption is not independent of the surface temperature and (1) must be generalized by including a temperature dependent surface absorption. This problem has been solved by Sparks and Loh [8], [9] by including a linearly temperature-dependent surface absorption. If the optical absorption $A$ is given by

$$
A=A_{o}(1+\alpha T)
$$

where $A_{o}$ is the Drude value of the surface absorption, $T$ is the surface temperature and $\alpha A_{o}$ is the measured temperature coefficient, then the required laser intensity $I$ in a pulse of duration $t_{p}$ required to raise the surface temperature of the metal to the melting value $T_{m}$ is given by

$$
\left(I t_{p}\right)_{m}=\frac{u}{\alpha A_{o}} \sqrt{t_{p} K C}
$$

where $u$ is a solution of the transcendental equation

$$
e^{u^{2}}\left[(1+\operatorname{erf}(u)]=\frac{T_{m}+1 / \alpha}{T_{o}+1 / \alpha} .\right.
$$




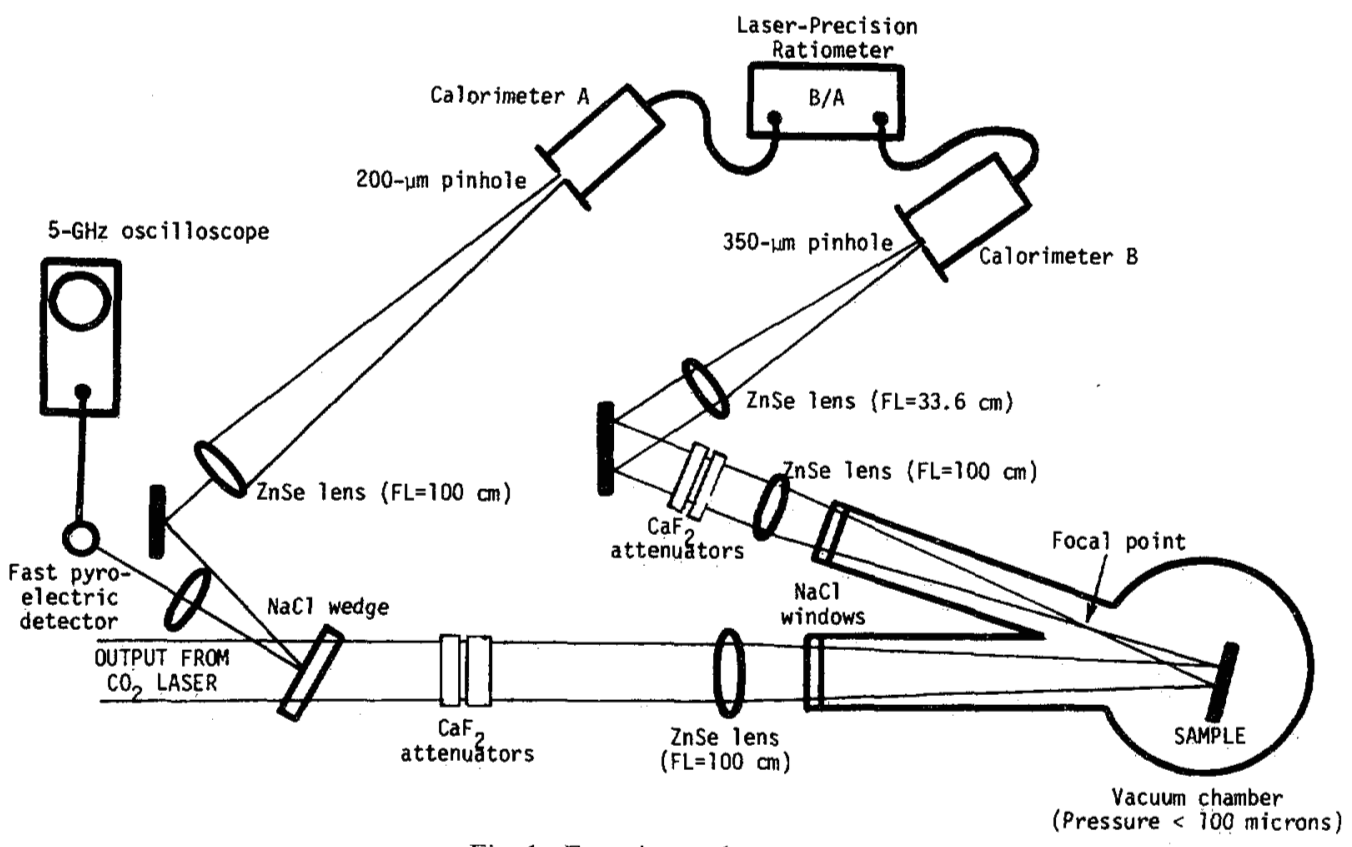

Fig. 1. Experimental apparatus.

Sparks and Loh derive similar expressions [9] for the threshold fluence required to vaporize the metal $\left(I t_{p}\right)_{V}$. Examination of (1) and (3) shows that the dependence of $\left(I t_{p}\right)$ on material parameters $K, C$, and $t_{p}$ is identical except for a multiplicative factor. The major difference is the $u / \alpha A_{o}$ factor which represents the temperature average from $T_{o}$ to $T_{m}$ of the $\left(T_{m}-T_{o}\right) /$ $2 A_{o}$ term of (1).

In order to determine the IR damage thresholds of several metals and to confirm the scaling trends and absolute predictions of (3), a series of measurements was undertaken for several pulse durations of $10.6 \mu \mathrm{m}$ radiation. For the test described in the following, the metal surfaces were irradiated in a vacuum with $10.6 \mu \mathrm{m}$ laser radiation. We have chosen to define the threshold of damage as that intensity where an observable, permanent change occurs in the surface when examined under $60 \times$ magnification with a white light source.

These tests were conducted on the Los Alamos Gigawatt Test Facility (GWTF), which produces a Gaussian pulse with duration variable from 1.7-60 ns (FWHM). The oscillator was grating-tuned to the $P(20), 10.59 \mu \mathrm{m}$ line. Both the oscillator and amplifier are UV-preionized TEA lasers. A two-stage electrooptic switch, driven by a laser-triggered spark gap provided the $1.7 \mathrm{~ns}$ pulse. A low-pressure smoothing tube was used in the oscillator cavity to provide a temporally smooth $58 \mathrm{~ns}$ output pulse [10]. The laser was operated at a pulse repetition frequency of $0.05 \mathrm{~Hz}$ so no appreciable heating of the surface was encountered.

A schematic of the damage test setup is shown in Fig. $\overline{1}$. The samples were placed $10 \mathrm{~cm}$ inside the focal point of a $1 \mathrm{~m}$ focal length lens where the $1 / e^{2}$ spot radius was $1 \mathrm{~mm}$. Peak fluence $\left(\mathrm{J} / \mathrm{cm}^{2}\right)$ at the sample plane was measured on each shot by use of a $200 \mu \mathrm{m}$-diameter pinhole located in the reference beam at the same distance from an identical lens. A Laser Precision Corporation ratiometer was employed to record the peak fluence of each laser shot, which was regulated with calibrated $\mathrm{CaF}_{2}$ and $\mathrm{BaF}_{2}$ attenuators. Prior to each test series, a calibration was performed with an identical pinhole centered at the sample plane, and the second channel of the ratiometer was used to measure the peak fluence. By this technique, damage plane fluence was determined on each shot to an accuracy of \pm 3 percent. A vacuum chamber with $\mathrm{NaCl}$ windows provided a background test environment of less than 100 mtorr. A liquid $\mathrm{N}_{2}$ cold trap prevented oil vapors from reaching the test sample. Single-shot damage thresholds were measured by irradiating the test sample at 25 different sites, at a fluence level which produced damage for approximately one half the shots. Samples were not cleaned prior to testing except to blow off dust particles.

$\mathrm{Cu}$ mirror substrates were 4 in diameter copper-plated aluminum-bronze, manufactured for use in the Antares ICF laser system. The copper plating was 0.040 in thick. Several vendors used standard optical fabrication techniques in polishing the test mirrors, while some were finished by single-point diamond turning at the DOE Y-12 facility. Additional samples of copper, molybdenum, and stainless steel 304 and aluminum 6061 mirrors were prepared by standard polishing techniques. The surface absorption $(1-R)$ was determined for each sample using a multipass arrangement with a $\mathrm{CO}_{2}$ laser and conventional calorimetry instrumentation. The measurement technique does not distinguish between absorption and low-angle scatter.

The single-shot normal-incidence damage thresholds for a variety of metals have been determined by the techniques described above. Table I shows the physical parameters of these materials, the measured value of the single-shot damage $F_{D}(O)$, the calculated melt threshold, the calculated vaporization threshold, and the melt thresholds using a constant surface absorption (1) from Sparks and Loh (where data are available).

The measured damage thresholds values range from a low of $0.34 \mathrm{~J} / \mathrm{cm}^{2}$ for polished aluminum to a high of $10 \mathrm{~J} / \mathrm{cm}^{2}$ for polished copper. When the measured thresholds are compared to the calculated thresholds with constant surface absorption (1), we see that the calculation consistently overestimates the 
TABLE I

SuMMary of DaMage Tests

\begin{tabular}{|c|c|c|c|c|c|c|}
\hline Material & Preparation & $1-R$ & $\left(\mathrm{It} \mathrm{p}_{\mathrm{p}}\right)_{\mathrm{m}} \mathrm{A}=$ const & $\left(I t_{p}\right)_{m}$ & $\left(I t_{p}\right)_{v}$ & $F_{D}(0)$ \\
\hline $\mathrm{Cu}$ & Polish & $.009 \pm .001$ & $15.8 \mathrm{~J} / \mathrm{cm}^{2}$ & $6.5 \mathrm{~J} / \mathrm{cm}^{2}$ & $9.1 \mathrm{~J} / \mathrm{cm}^{2}$ & $10.7 \pm .5 \mathrm{~J} / \mathrm{cm}^{2}$ \\
\hline Moly & Polish & .019 & 8.5 & - & - & $5.3 \pm .3$ \\
\hline $55-304$ & Polish & .102 & 0.56 & - & . & $0.87 \pm .08$ \\
\hline AL6061 & Polish & .104 & 2.7 & 1.8 & 4.56 & $0.34 \pm .02$ \\
\hline AL6061 & SPDT & .021 & 2.7 & 7.8 & 4.56 & $0.62 \pm .04$ \\
\hline
\end{tabular}

\footnotetext{
$\left(I t_{p}\right)_{m}$ a $A=$ const melt threshold for constant absorption

$\left(I t_{p}\right)_{m}$ melt threshold for variable absorption

(It $\left.t_{p}\right) \mathrm{v}$ vaporization threshold.
}

damage threshold. When the measurements for $\mathrm{Cu}$ are compared to the theory of Sparks and Loh, we see that the observed threshold is consistent with the vaporization threshold rather than the melt threshold [9]. The comparison for aluminum between measurement and calculation is very poor, most likely due to the formation of surface oxides which can dramatically modify the surface absorption [11]. For stainless steel and molybdenum, the observed damage and the calculated melt thresholds differ by 35 and 60 percent, respectively, in opposite directions. Including the correction for a temperature dependent surface absorption will lower the calculated damage threshold. This will improve the agreement with the molybdenum data, but will enhance the disagreement with the stainless steel data.

In order to confirm the predicted pulse width dependence of the damage fluence of (1) and (3), the laser was adjusted for long-pulse operation by rotating the middle polarizer of the two-stage Pockels cell to allow the entire oscillator pulse to pass through the amplifier, giving a pulse duration of $58 \pm 7 \mathrm{~ns}$. In general, the 58 ns pulses were asymmetric, containing a sizable fraction of the energy in a long $(150 \mathrm{~ns})$ tail. The value quoted is the average FWHM of five typical pulses. The measured damage thresholds for these two pulse durations are listed in Table II and plotted in Fig. 2 for the same mirror samples described above. Also shown in the figure is the trend line for $F_{D}(O)$ proportional to $\left(t_{p}\right)^{1 / 2}$. As is evident from this figure, the damage fluence scales as $\left(t_{p}\right)^{1 / 2}$ in agreement with (1) and (3) and confirming the earlier results of Saito et al. [12].

Single-shot damage thresholds are of interest in determining the physical processes involved in absorption at metal surfaces; however, they do not fully describe the operating constraints faced by a metal mirror when used as an optical element in a high-power laser system. Moreover, as was shown above, the variety of damage phenomenon makes the determination of a unique damage threshold extremely difficult. Hence, we have chosen to define damage by imposing a definite performance criterion on the optical element under test and then measure the element's progressive performance degradation as the surface is damaged by repeated exposure to a high intensity laser beam.

For the performance criterion, we have chosen to measure the peak brightness of the beam reflected from the mirror sur-
TABLE II

\begin{tabular}{lcc}
\hline Material & $F_{D}(0) @ 7.7 \mathrm{~ns}$ & $F_{D}(0) \oplus 58 \mathrm{~ns}$ \\
\cline { 2 - 3 } & $10.1 \pm .5 \mathrm{~J} / \mathrm{cm}^{2}$ & $67 \pm 3 \mathrm{~J} / \mathrm{cm}^{2}$ \\
Molybdenum & $5.3 \pm .3$ & $25 \pm 1$ \\
Stainless Stee & $.87 \pm .08$ & $4.6 \pm .5$ \\
\hline
\end{tabular}

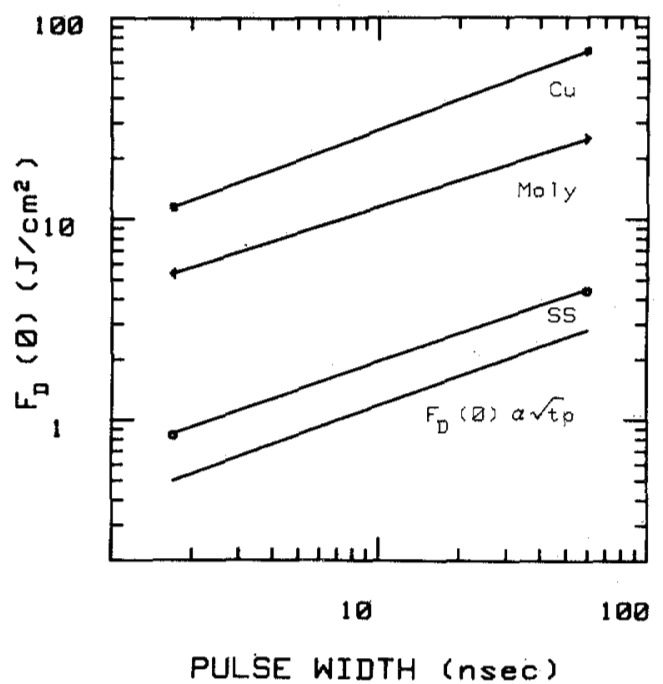

Fig. 2. Damage thresholds for $\mathrm{Cu}$, molybdenum, and stainless steel 304 for $1.7 \mathrm{~ns}$ pulses and $58 \mathrm{~ns}$ pulses of $10.6 \mu \mathrm{m}$ light in vacuum. Solid curve shows expected $\sqrt{t_{p}}$ dependence on pulse duration.

face. This is the actual criterion of importance in any highpower/high-brightness laser system and can be directly measured by standard laboratory techniques. An experimental arrangement similar to that described above was employed (see Fig. 1). A $10.6 \mu \mathrm{m}$ laser beam was converged toward a mirror surface in vacuum and the reflected signal was passed through a spatial filter where the peak brightness of the beam was measured. For these measurements the mirror was tilted $15^{\circ}$ to allow access to the reflected beam. At a fixed fluence level, the beam brightness of the reflected beam was then measured as a function of the number of shots at a fixed location on the mirror surface. For this test both diamond-turned 
and conventionally polished substrates as described above were used. Figs. 3 and 4 show some typical results for $\mathrm{Cu}$ mirrors with conventionally polished surfaces (Fig. 3) and with diamond-turned surfaces (Fig. 4). Beam brightness is plotted versus shot number for several values of incident fluence normalized to the single-shot damage fluence, $F_{o}$ for the mirror under test. For both the diamond-turned and conventionally polished mirrors the beam brightness decreases with shot number. The rate of brightness degradation is initially fast and then slows as is evident from the slope change after $\sim 40$ shots. These data show that for operation at one half of the single shot damage level, the mirror lifetime is essentially infinite. For increased values of the irradiating fluence, the brightness of the reflected beam degrades, indicating a tradeoff between operating fluence and mirror lifetime. It should be noted that if the laser fluence is decreased to $\sim F_{o} / 100$, the brightness returns to 100 percent, indicating that the brightness degradation is a dynamic effect at the mirror surface. Separate scanning electromicrographs of the surface show this degradation to be due to the production of a fine-scale $(1 \mu \mathrm{m})$ surface structure [13].

With this brightness data, lifetime curves can be constructed. Fig. 5 shows such a lifetime curve for $\mathrm{Cu}$ mirrors in vacuum where the number of shots required to produce a 10 percent reduction in beam brightness is plotted as a function of the beam fluence for all of the mirror samples tested. Input fluences have been normalized to the measured single-shot fluence, $F_{o}$. The data from all vendors scatter about an exponential trend line. The data can be fit by the function

$$
N=10^{7\left(1-F / F_{O}\right)}
$$

where $N$ is the number of shots at fluence $F$ required to reduce the beam brightness to 0.9 of its starting value, and $F_{o}$ is the single-shot damage threshold. The prefactor 7 is determined by a least-squares fit to the data. The universality of the trends among polishers suggest that the failure mode is dominated by intrinsic properties of the $\mathrm{Cu}$ (e.g., melt levels or vaporization levels) rather than details of the mirror preparation technique. If one defines 10 percent brightness loss as the maximum acceptable beam degradation, then (5) requires operation at 71 percent of single-point damage levels for 100 shot-lifetimes and operation at 57 percent of single-point damage levels for 1000 shot-lifetimes. Note that all lifetime tests to date have been done with no more than 100 shots. The estimates beyond that level are strictly extrapolations.

In this discussion we have made a first attempt at describing long-term damage phenomena in metal surfaces. The use of the 90 percent peak brightness level as a damage criteria is arbitrary, but does provide a simple interpretation of the experimental results that is independent of polishing techniques. Different damage criteria will lead to different conclusions. In particular, if larger beam brightness degradations are allowed, much more detail appears in the lifetime data and the conventionally polished mirrors may show a long-term lifetime advantage over the diamond-turned mirrors. These details will be discussed in subsequent reports.

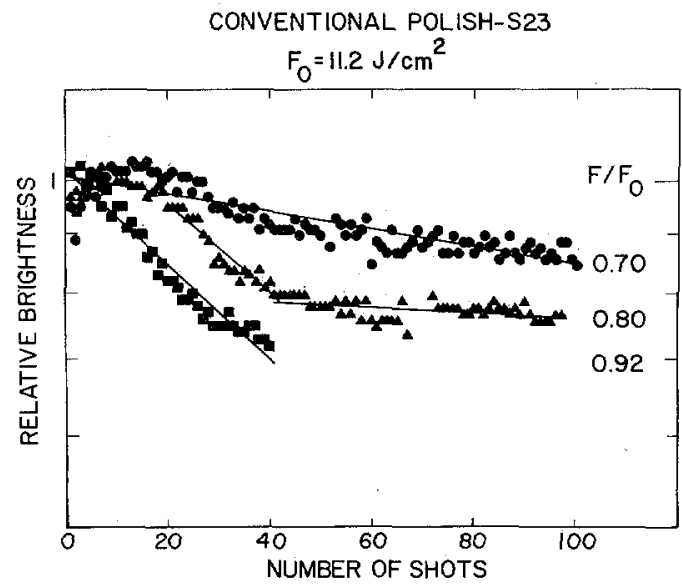

Fig. 3. Brightness degradation of a conventionally polished $\mathrm{Cu}$ mirror in vacuum when illuminated with successive laser pulses at a fixed peak fluence level. Pulse duration $1.7 \mathrm{~ns}, P(20), 10 \mu \mathrm{m}$ radiation.

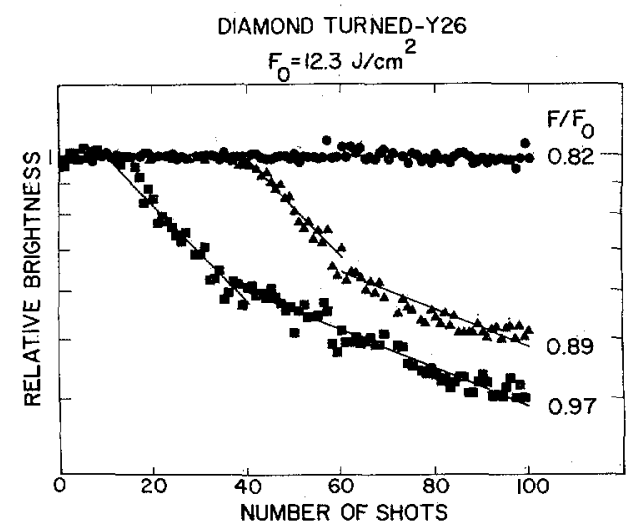

Fig. 4. Brightness degradation of single-point diamond turned $\mathrm{Cu}$ mirror in vacuum when illuminated with successive laser pulses at a fixed peak fluence level. Pulse duration $1.7 \mathrm{~ns}, P(20), 10 \mu \mathrm{m}$ radiation.

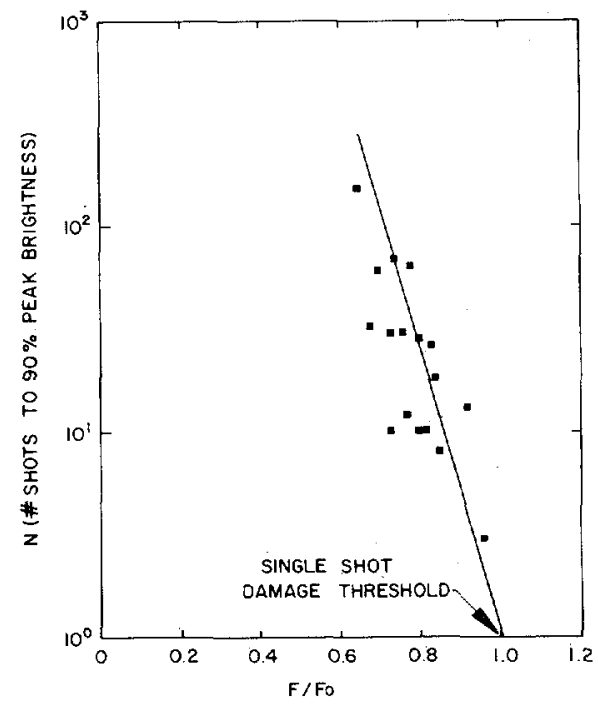

Fig. 5. Mirror lifetime curve for conventionally polished and micromachined $\mathrm{Cu}$ mirrors for various fixed values of the irradiating fluence $F$, normalized to the single point damage fluence $F_{o}$. Lifetime is defined as the number of shots required to reduce the beam brightness to 90 percent of its initial value. Laser pulse is $1.7 \mathrm{~ns}$ at $10.6 \mu \mathrm{m}$ 


\section{Grazing Incidence Damage Thresholds}

In the damage threshold measurements described above, the laser beam was chosen to be normal to the metal surface under test. In this case the two components of electric field vector, $E_{s}$ (field perpendicular to the plane of incidence) and $E_{p}$ (field in the plane of incidence) both are parallel to the metal surface and the absorption experienced by the incident light field is independent of polarization. For the more general case of nonnormal incidence, differences in the surface absorption arise for $E_{s}$ and $E_{p}$ radiation. Recently, Mumola [14], and earlier, Goldstein et al. [15], have pointed out that this effect could be used to substantially increase the damage threshold of metal mirrors when used at grazing angles of incidence. In this section we will discuss a model for grazing incidence damage to metal surfaces and will describe the results of experimental tests of this theory.

For a metal described by a complex index of refraction $\hat{n}$ where

$$
\hat{n}=n+i K
$$

the intensity reflection coefficient of a light wave at an angle of incidence $\Theta$ is given by [16]

$$
\begin{aligned}
& R_{p}=\frac{\left(n^{2}+k^{2}\right) \cos ^{2} \Theta-2 n \cos \Theta+1}{\left(n^{2}+k^{2}\right) \cos ^{2} \Theta+2 n \cos \Theta+1} \\
& R_{s}=\frac{\left(n^{2}+k^{2}\right)-2 n \cos \Theta+\cos ^{2} \Theta}{\left(n^{2}+k^{2}\right)+2 n \cos \Theta+\cos ^{2} \Theta}
\end{aligned}
$$

in the limit where $\left(n^{2}+k^{2}\right) \gg 1$ and where $R_{p}$ and $R_{s}$ are the intensity reflection coefficients for radiation polarized parallel and perpendicular to the plane of incidence, respectively.

In order to make (6) and (7) more transparent, we can use the following approximations. For most metals in the IR $n \gg 1$ and $k \gg 1$ and (6) and (7) can be further simplified by expanding for the denominator in powers of $2 n \cos \Theta /$ $\left(n^{2}+k^{2}\right)$ to produce

$$
\begin{aligned}
& R_{p}=1-\frac{4 n}{\left(n^{2}+k^{2}\right) \cos \Theta} \\
& R_{s}=1-\frac{4 n \cos \Theta}{n^{2}+k^{2}} .
\end{aligned}
$$

If we define in analogy with (2) an angular dependent absorption coefficient $A(\Theta)$ by

$$
A(\Theta)=1-R(\Theta)
$$

then we have from (8) and (9)

$$
\begin{aligned}
& A_{p}(\Theta)=A(O) / \cos \Theta=A_{o}(1+\alpha T) / \cos \Theta \\
& A_{s}(\Theta)=A(O) \cos \Theta=A_{o}(1+\alpha T) \cos \Theta
\end{aligned}
$$

where the normal incidence absorption is given by $A(O)$ and

$$
A(O)=\frac{4 n}{n^{2}+k^{2}}
$$

For a metal surface at an angle of incidence $\Theta$ to an incoming laser beam, the calculation for the surface damage threshold must be modified in two respects. First, the surface fluence must be reduced from the normal beam fluence $I$, by the factor $\cos \Theta$ due to the increased surface area now irradiated by the rotated laser beam. Secondly, the absorption, $A(\Theta)$, must be modified by the complex Fresnel equations above to include electric field components both parallel and perpendicular to the metal surface. If we assume that $A_{o}$ and $\alpha$ are not functions of $\Theta$ then the expression for the damage threshold for surface melting of (1) or (3) can be corrected for nonnormal incidence using (11) and (12) and corrected for surface fluence to obtain

$$
\begin{aligned}
& \left(I t_{p}\right)_{m, p}=\frac{u}{\alpha A_{o}} \sqrt{t_{p} K C} \\
& \left(I t_{p}\right)_{m, s}=\frac{u}{\alpha A_{o} \cos ^{2} \Theta} \sqrt{t_{p} K C}
\end{aligned}
$$

where $p$ and $s$ refer to parallel and perpendicular polarization of the radiation, $I$ is the beam fluence normal to beam direction (this causes an extra $\cos \Theta$ to appear on the RHS above). Note that $u / \alpha A_{o} \sqrt{t_{p} K C}$ is just the measured value of the damage fluence at normal incidence tabulated in Table I, i.e., $F_{o}(O)$. For $p$-plane polarization (14), the damage threshold remains constant, independent of incident angle (for large angles the approximations used in deriving (8) and (9) fail and the damage threshold does not increase for angles $>80^{\circ}$ ). For $s$-plane polarization (15), the damage fluence increases as $\left(\cos ^{2} \Theta\right)^{-1}$ and at large angles $\left(\Theta>80^{\circ}\right)$ will show a dramatic increase over the normal incidence value.

In order to experimentally verify these predictions a series of single-shot damage tests were performed in a vacuum for the same four materials used above, $\mathrm{Cu}$, stainless steel 304, molybdenum, and aluminum. The experimental setup was previously described except that the mirror surfaces were mounted on a rotation stage to allow rotation from $\Theta=0^{\circ}$ to $\Theta=85^{\circ}$. Pulse durations of $1.7 \mathrm{~ns}$ were used for these tests. The maximum angles used were determined by the maximum available laser fluence $\left(\sim 50 \mathrm{~J} / \mathrm{cm}^{2}\right)$. With this setup single-point damage thresholds were established by the same techniques described for the normal incidence tests. The results of these tests for $s$-plane radiation are shown in Fig. 6 . The value of the damage fluence plotted is the fluence normal to the incidence beam. Also shown on the figure are the predicted damage thresholds calculated from (7) and (12) using the material parameters of Table I. In general the agreement between the measured and calculated values is good. In particular the $\cos ^{2} \Theta$ dependence in the damage threshold is well borne out by the experimental data. The largest increase in the damage level measured was that for stainless steel where the threshold increased from 0.6 $\mathrm{J} / \mathrm{cm}^{2}$ to $24 \mathrm{~J} / \mathrm{cm}^{2}$ at an $80^{\circ}$ incidence angle.

\section{CONClusion}

In this paper we have described the results of damage tests on metal mirrors with short-pulse $\mathrm{CO}_{2}$ laser radiation. Singlepoint damage thresholds were determined for copper, stainless steel, molybdenum, and aluminum mirrors. 


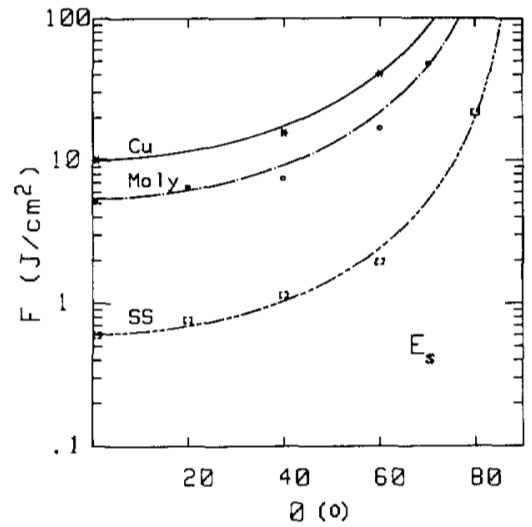

Fig. 6. Grazing incidence damage thresholds $F$, in vacuum for $1.7 \mathrm{~ns}$ pulses of $10.6 \mu \mathrm{m}$ tadiation polarized perpendicular to the incident plane $\left(E_{S}\right)$ for various angles of incidence $\Theta$. Solid curves are calculated from (7). Data for $\mathrm{Cu}$, molybdenum, and stainless steel 304 are shown.

Lifetime tests of $\mathrm{Cu}$ mirrors in vacuum at the 100 shot level have shown consistent results among a variety of manufacturers and manufacturing techniques. Lifetime depends exponentially on the normalized fluence levels at the mirror surface. For 1000 shot lifetimes, operation at $\sim 50$ percent of the singlepoint damage level is required to prevent degradation of the optical quality of the reflected beam.

Finally, dramatic improvements in the damage thresholds for metal mirrors were obtained at grazing incidence for the first time in the IR. The experimental results were compared to model calculations and were found to be in general agreement for radiation polarized parallel to the metal surface.

\section{ACKNOWLEDGMENT}

The authors would like to thank C. A. Fenstermacher, C. R. Phipps, B. E. Newnam, P. J. Mumola of Perkin Elmer Corporation, and R. V. Kung of W. J. Schafer Associates for many helpful discussions. We would especially like to thank N. Kurnit for providing the surface absorption measurements. The technical assistance of R. F. Harrison is also gratefully acknowledged.

\section{REFERENCES}

[1] B. E. Newnam and D. H. Gill, "Damage resistance of $\mathrm{CO}_{2}$ fusion laser optics," Opt. Eng., p. 579, Nov./Dec. 1979.

[2] J. J. Hayden and I. Liberman, "Measurements of $10.6 \mu \mathrm{m}$ damage thresholds in $\mathrm{Ge}, \mathrm{Cu}, \mathrm{NaCl}$, and other optical materials at levels up to $10^{10} \mathrm{~W} / \mathrm{cm}^{2}$ " in Laser Induced Damage in Optical Materials, 1976, A. J. Glass and A. H. Guenther, Eds. Washington, DC: Nat. Bur. Stand., publication 462, 1977, p. 173.

[3] S. J. Thomas, C. R. Phipps, Jr., and R. F. Harrison, "Optical damage limitations for copper mirrors used in $\mathrm{CO}_{2}$-ICF laser systems," in Laser-Induced Damage in Optical Materials: 1980 , H. E. Bennett, A. J. Glass, A. H. Guenther and B. E. Newnam, Eds. Washington, DC: Nat. Bur. Stand., to be published, p. 173.

[4] J. F. Ready, "Change of reflectivity of metallic surfaces during irradiation by $\mathrm{CO}_{2}$ TEA laser pulses," IEEE J. Quantum Electron., vol. QE-12, p. 137, 1976.

[5] J. O. Porteus, D. L. Decker, D. J. Grandjean, S, D. Seitel, and W. W. Faith, "Defect-damage-resistant copper mirrors," in Laser Induced Damage in Optical Materials: 1979, H. E. Bennett, A. J. Glass, A. H. Guenther and B. E. Newnam, Eds. Washington, DC: Nat. Bur. Stand., publication 568, 1980, p. 175.
[6] H. S. Carslaw and J. C. Jaeger, Conduction of Heat in Solids. New York: Oxford, 1959, 2nd ed., p. 75.

[7] M. Sparks, "Theory of laser heating of solids," I. Appl. Phys, vol. 47 , p. $837,1976$.

[8] M. Sparks and E. Loh, Jr., "Temperature dependence of absorption in laser damage of metallic mirrors: I, melting," J. Opt. Soc. Amer., vol. 69, p. 847, 1979.

[9] _- "Temperature dependence of absorption in laser damage of metallic mirrors: II. Vaporization and heating the vapor," $J$. Opt. Soc. Amer., vol. 69, p. 859, 1979.

[10] J. F. Figueira and A. V. Nowak, "Carbon dioxide laser system with zero small-signal gain," Appl. Opt., vol. 19, p. 420, 1980.

[11] J. T. Cox and G. Hass, "Aluminum mirrors $\mathrm{Al}_{2} \mathrm{O}_{3}$ protected with high reflectance at normal but greatly decreased reflectance at higher angles of incidence in the 8-12 $\mu \mathrm{m}$ region," Appl. Opt., vol. 17, p. 333,1978 .

[12] T. T. Saito, D. Milam, P. Baker, and G. Murphy, "1.06 $\mu \mathrm{m}, 150$ ps laser damage study of diamond turned/polished and polished metal mirrors," in Laser Induced Damage in Optical Materials: 1975. Washington, DC: Nat. Bur. Stand., special publication, 435,1975 , p. 29.

[13] J. F. Figueira and S. J. Thomas, "Observation of the morphology of laser-induced damage in copper mirrors," Appl. Phys. Lett., vol. 40 , p. 260,1982

[14] P. B. Mumola, J. J. Ewing, W. T. Leland, and C. A. Fenstermacher, "Advanced $\mathrm{CO}_{2}$ laser fusion driver concepts," in Proc. Int. Conf. Lasers ' 80 , V. Corkrun, Ed., to be published.

[15] I. Goldstein, D. Bua, and F. A. Horrigan, "Pulsed laser damage to uncoated metallic reflectors," in Laser Induced Damage in Optical Materials: 1975. Washington, DC: Nat. Bur. Stand., special publication, 435, 1976.

[16] M. Born and E. Wolf, Principles of Optics. New York: Pergamon, p. $615-624,5$ th ed.

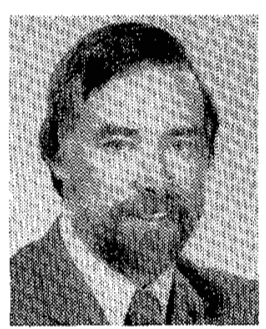

Joseph F. Figueira was born in Paris, IL, on March 7, 1943. He received the B.S. degree in engineering physics and mathematics from the University of Illinois, Urbana, in 1965, and the M.S. and Ph.D. degrees in physics from Cornell University, Ithaca, NY, in 1971.

He has been a Staff Member at the Los Alamos National Laboratory, University of California, Los Alamos, NM, since 1971, working in the area of laser development in support of the Inertial Confinement Fusion Program and the Molecular Laser Isotope Separation Program. In 1976 he was appointed Group Leader of the Laser Research and Development group. His current interests include UV and IR gas laser technology, nonlinear optics, laser interaction with matter, and advanced laser science and technology.

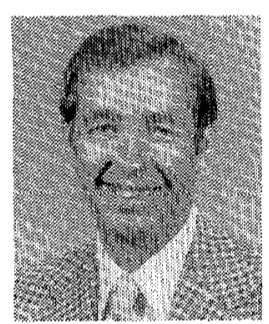

Scott J. Thomas was born in Spruce Pine, NC, on November $18,1934$.

He joined the U.S. Air Force in 1955 and worked as an Aircraft Technologist in the Strategic Air Command. From 1961-1974 he was employed by the Lawrence Livermore Laboratory, Livermore, CA, in the Laser Fusion Division. He joined the Los Alamos National Laboratory, University of California, Los Alamos, NM, in 1974, and worked in laser research and development in the Laser Fusion Program until 1981. From 1981 to present he has worked in the Applied Photochemistry Division. He has published work on laser produced plasmas, laser photochemistry, chemical lasers, dye lasers, gas lasers, nonlinear optical studies, and laser damage to optical surfaces. His present position is a Staff Member working in laser research and development. 